\title{
Protocol for a Diagnostic Accuracy Study of Polymerase Chain Reaction for Detecting Group B Streptococcus Colonisation in Early Labour or with Spontaneous Ruptured Membranes
}

\author{
Laura Cunningham ${ }^{1}$, Bradley de Vries ${ }^{2,3}$, Jon A. Hyett ${ }^{2,3}$, Hala Phipps ${ }^{4,5}$ \\ ${ }^{1}$ Royal Prince Alfred Hospital, Sydney, Australia \\ ${ }^{2}$ RPA Women \& Babies, Royal Prince Alfred Hospital, Sydney, Australia \\ ${ }^{3}$ Discipline of Obstetrics, Gynaecology and Neonatology, University of Sydney, Sydney, Australia \\ ${ }^{4}$ Sydney Local Health District, Sydney, Australia \\ ${ }^{5}$ Faculty of Nursing and Midwifery, University of Sydney, Sydney, Australia \\ Email: *laura.cunningham2@health.nsw.gov.au
}

How to cite this paper: Cunningham, L., de Vries, B., Hyett, J.A. and Phipps, H. (2018) Protocol for a Diagnostic Accuracy Study of Polymerase Chain Reaction for Detecting Group B Streptococcus Colonisation in Early Labour or with Spontaneous Ruptured Membranes. Open Journal of Obstetrics and Gynecology, 8, 1023-1031. https://doi.org/10.4236/ojog.2018.811103

Received: August 20, 2018

Accepted: September 17, 2018

Published: September 20, 2018

Copyright $\odot 2018$ by authors and Scientific Research Publishing Inc. This work is licensed under the Creative Commons Attribution International License (CC BY 4.0).

http://creativecommons.org/licenses/by/4.0/

\section{(c) (i) Open Access}

\begin{abstract}
Background: Group B Streptococcus [GBS] is a bacterium which transiently colonises the genital tract and can be transmitted from mother to baby at birth. Babies colonised with GBS can develop early-onset group B streptococcus disease [EOGBSD] which can lead to extended hospital stay, disability and death. One of the primary methods for determining which women are most likely to be GBS positive at the time of birth is antenatal universal culture-based screening. Recently Polymerase Chain Reaction [PCR] screening has emerged as a point-of-care method for screening women during the intrapartum period. This study will compare the diagnostic accuracy of this new technology and antenatal culture-based screening at 35 to 37 weeks gestational age, with the reference standard of formal culture-based testing in labour. Methods: This prospective observational study will take place in an Australian hospital. Consecutive women with one or more live fetuses, intending to have a vaginal birth will be asked to participate. Planned screening for GBS colonisation using microbiological culture on a self-collected specimen will occur at 35 to 37 completed weeks gestational age as per our usual hospital policy. A PCR GBS test by Xpert GBS (Cepheid) will be performed on admission to labour ward or at the time of rupture of membranes. The reference standard will be a formal GBS culture on a combined lower vaginal and perianal swab. The sensitivity, specificity, positive and negative predictive values, and positive and negative likelihood ratios will be estimated for both
\end{abstract}


antenatal screening and the intrapartum Xpert GBS (Cepheid) point-of-care test and compared to the reference standard. Results: It is expected that the study will be completed by mid to late 2020. Conclusion: This study has the potential to improve the accuracy of GBS screening of pregnant women and therefore health outcomes for mothers and babies. There is also the potential for a cost savings to the health system.

\section{Keywords}

GBS Screening, Group B Streptococcus, Antibiotic Prophylaxis, Early-Onset Group B Strep, Intrapartum, Rapid Diagnostic Test, Protocol, Neonates

\section{Background}

Antimicrobial resistance is a major threat to human health worldwide [1]. Antibiotic overuse is the leading cause of antibiotic resistance. Up to one-half of antibiotic use in humans is either unnecessary or inappropriate [2] [3]. One means of countering that threat is through "antibiotic stewardship", ensuring that antibiotics are used when they are needed and not otherwise. However, accurate antibiotic administration requires accurate diagnosis [1].

GBS is an asymptomatic and transient bacterium that colonises the lower genital tract and/or gastrointestinal tract of $10 \%-40 \%$ pregnant women [4]. The global mean prevalence of maternal GBS colonisation is $17.9 \%(11.1 \%-22.4 \%)$ [5]. In Australia it is estimated that $23 \%$ of pregnant women are GBS positive [6]. Colbourn et al. [7] found that without intervention, $33 \%$ of babies born to GBS positive mothers will become GBS colonised and of those babies, 1 in 33 will develop early-onset Group B Streptococcus disease [EOGBSD]. Jeffery and Lahra [8] state that in Australia the natural incidence of blood culture-positive EOGBSD is 1.4/1000 live births. Where it occurs, EOGBSD can be severe. Its complications can include septicaemia, pneumonia, meningitis, long-term disability and death. Accordingly, to prevent transmission, all Australian hospitals are required to adopt one of two available GBS screening methods for pregnant women [9]. Those are universal culture-based screening and risk-factor screening. Another method, real-time PCR screening by Xpert GBS, is yet to be studied or used for GBS screening in Australia.

\subsection{GBS Screening Methods}

\subsubsection{Universal GBS Culture-Based Screening}

Universal culture-based screening requires a combined lower vaginal and perianal swab from all pregnant women at 35 - 37 weeks gestation. Women identified as GBS positive are offered prophylactic antibiotics from rupture of membranes until the birth of their baby. In settings that use this form of screening the incidence of EOGBSD is 0.23/1000 (CI 0.13-0.59) [5]. Culture-based screening is an accurate method of detecting GBS at the time the swab is taken. But because 
GBS is transient, a swab at 35 - 37 weeks can be an imperfect predictor of GBS status at birth. The negative predictive value of an antenatal GBS culture declines if the interval between screening and birth is $\geq 5$ weeks [10]. As a consequence, an estimated $12 \%-13.6 \%$ of women receive antibiotics in labour for GBS when they are GBS negative at the time they give birth [11]. While the Centres for Disease Control and Prevention [10] reports that $60 \%$ of babies who develop EOGBSD are born to mothers who tested GBS negative at 35 - 37 weeks gestation.

\subsubsection{Risk-Factor Screening}

Risk-factor screening is the method used in the United Kingdom as well as some Australian hospitals. Risk-factor screening requires antibiotics to be offered to women with the following risk factors: preterm labour; rupture of membranes $\geq$ 18 hours; maternal fever $\geq 38$; a previous infant with EOGBS; GBS bacteriuria during the current pregnancy; previous GBS-positive swab in current pregnancy; confirmed chorioamnionitis; and, other twin with current EOGBSD [9]. The main concern with risk-factor screening is timely identification of the risk so that antibiotics can be administered [12]. The incidence of EOGBSD in settings that use this method of screening is $0.5 / 1000$ live births [13].

\subsubsection{Point-of-Care PCR Screening: New Technology}

Since GBS screening was introduced in the 1990s, PCR screening has emerged and promises greater accuracy for identifying GBS status at time of birth. El Helali et al. [14] have shown that, using one PCR device, Xpert GBS (Cepheid), screening of pregnant women can be undertaken by trained midwives in labour ward. The process involves the midwife taking a lower vaginal swab when a woman is admitted to labour ward and processing this in the PCR machine within the labour ward with a result available in less than 50 minutes [14]. GBS-positive mothers can then receive prophylactic antibiotics as they would in the universal screening approach. The Xpert GBS (Cepheid) Assay had a sensitivity of $98.5 \%$ and a specificity of $99.6 \%$ for detecting GBS status at birth when compared to antenatal culture [14]. The Xpert GBS (Cepheid) has the potential to reduce the number of babies developing EOGBSD as well as antibiotic stewardship as it relates to maternal GBS screening and treatment. This new form of screening has since been introduced at various hospitals in Europe and has been found to be cost-neutral when compared to universal antenatal culture-based screening [15]. Further research is needed into the accuracy and cost-effectiveness of point-of-care PCR screening for GBS in different settings. Before this PCR screening can be introduced in Australia, the findings [14] need to be shown to be generalizable in an Australian population.

\section{Objectives}

\subsection{Aims}

To assess the accuracy of point-of-care Xpert GBS (Cepheid) near the time of 
birth and antenatal culture-based screening at 35 to 37 weeks gestational age.

\subsection{Hypothesis}

1) Point-of-care Xpert GBS (Cepheid) in early labour has a sensitivity of at least $95 \%$ and specificity of at least $95 \%$ for diagnosing or excluding maternal colonisation with GBS compared with a combined lower vaginal and perianal culture in early labour.

2) Point-of-care Xpert GBS (Cepheid) has improved sensitivity and specificity compared with culture-based screening at 35 to 37 weeks gestational age.

\section{Methods}

\subsection{Study Design}

This will be a prospective observational study.

\subsection{Participants}

Eligibility criteria are women with an ongoing viable pregnancy planning to have a vaginal birth at Royal Prince Alfred Hospital, Sydney and who are approximately 34 weeks' gestational ages.

Exclusion criteria are to be applied at $36^{+0}$ completed weeks gestational age. They include: fetal death in utero; planned caesarean section; informed consent not obtained; and, age less than 18 years.

Potential participants will be approached in the antenatal clinic from $34^{(+0)}$ weeks gestational age onwards, and term women in labour ward/ birth centre (on admission if appropriate). Consecutive women in the public health care system will be approached.

\subsection{Test Methods}

\subsubsection{Index Test}

The index test will be performed by clinical staff caring for the participants in early labour. We are yet to finalise the logistics of the PCR technology during the study. Staff will be unaware of the results of the reference standard test. However, they will be aware of the results of the low vaginal and perianal swab and culture for GBS routinely performed at 35 to 37 weeks gestational age.

The Xpert GBS (Cepheid) is an automated process that integrates DNA extraction, amplification and detection without the need for enrichment. The process is completed in less than 50 minutes and the DNA target sequence is within the 3 DNA area adjacent to the streptococcus agalactiae $c f b$ gene [14]. Each single-use cartridge contains a processing control for the sample as well as an internal control. PCR data from a sample is collected by a computer that then detects and reports GBS status [14]. We are yet to decide what the Xpert GBS (Cepheid) cycle threshold will be for determining GBS colonisation of a sample. Cepheid has confirmed that the primers and probe sequences used in the assay cannot be provided as they are proprietary information. 


\subsubsection{Reference Standard}

The reference standard will be a combined lower vaginal and perianal swab collected by a doctor or midwife within 24 hours of birth.

GBS swabs are first inoculated in an enrichment medium (LIM broth) and after 18 - 24 hours incubation are cultured on a Chromogenic GBS plate (looking for colour/typical colonies). At this stage negative samples are excluded and some positive samples go through a final identification confirmation. Laboratory staff will be unaware of the index test results.

\subsubsection{Analysis}

The sensitivity, specificity, positive and negative predictive values will be calculated by constructing a $2 \times 2$ table comparing the reference standard with the index test. Positive and negative likelihood ratios will be calculated from the sensitivity and specificity. Sensitivity and specificity will be reported as percentages with 95\% confidence intervals. The diagnostic accuracy of the index test (point-of-care Xpert GBS) will be compared with that of screening at 35 to 37 weeks gestational age using McNemar's test to compare proportions. We do not expect indeterminant tests for the reference standards. If any occur, the woman will be excluded but the number will be reported. Indeterminant index tests will be included in the study and a sensitivity analysis will be performed assuming they are all positive or all negative. Participants with missing data for the reference standard will be excluded. At this stage we are not sure if index tests that are classified as GBS status unknown will be analysed as if they are positive or screened via a risk-factor approach. There is no planned analysis of variability of diagnostic accuracy within subgroups or participants.

\subsubsection{Sample Size}

Assuming the true sensitivity is $98 \%$, for a desired precision of $\pm 2 \%$, with an assumed $10 \%$ loss to follow-up, 210 participants with a positive reference standard will be required. Assuming a true specificity of $99.6 \%$ and a desired precision of $\pm 1.6 \%, 67$ participants with a negative reference standard will be required. Assuming $20 \%$ of all participants will have a positive reference standard (GBS carriage rate), 1050 women will need to be recruited.

For comparing point-of-care Xpert GBS (Cepheid) with culture-based screening at 35 to 37 weeks gestational age we assumed a sensitivity of $90 \%$ for culture-based screening [11] and 95\% for the point of care test. For a $20 \%$ rate of GBS colonisation, power of $80 \%$, and alpha value of $5 \%, 1155$ participants will be required [16].

Thus, to meet all aims, 1155 participants will be required.

\section{Outcomes}

\subsection{Primary Outcomes}

Specificity, sensitivity, positive and negative predictive values, positive and negative likelihood ratios will be also calculated. 


\subsection{Secondary Outcomes}

Maternal outcomes to be collected: maternal age; parity, gestational age at delivery; type of labour onset; duration of labour; duration of membrane rupture before delivery; intrapartum fever; GBS status at delivery; antibiotic prophylaxis or antibiotic therapy; number of doses of penicillin (or other); previous child with early-onset GBS disease; GBS screening results and incidence; bacteriuria during the current pregnancy, mode of delivery, maternal admission to the Intensive Care Unit, maternal death (death while pregnant) [17].

Neonatal outcomes to be collected: resuscitation; admission to Neonatal Intensive Care Unit; Apgar scores at 5 minutes; infant birth weight; length of infant hospital stay; suspected EOGBDS; confirmed bacteraemia EOGBSD; antibiotic administration and number of doses; neonatal death; neonatal disability (death before 28 completed days after live birth) [17].

\section{Recruitment}

\section{Consent}

Consent will occur at two possible time points:

a) Antenatally

b) In the early phase of labour

Women will be approached at the time of their usual antenatal clinic appointment at any time from 30 weeks' gestation, but aiming for 34 weeks. Recruitment will occur at one major tertiary hospital in Australia. Strategies to maximise recruitment will include:

- Midwives and doctors will receive a short training session about the study so that they feel confident to discuss the study with pregnant women.

- Participants will be provided with written information via information pamphlets, posters, the development of an App with information about GBS and the study will be considered.

- The presence of a recruitment midwife in the antenatal clinics who will provide information about the study and obtain informed consent.

- Each participant will be given the contact details of the research midwife who will be able to answer any queries or concerns she may have about the trial.

\section{Data Collection and Management}

\subsection{Data Collection Methods}

Required data fields will be collected and/or extracted from both medical records and electronic obstetric database.

\subsection{Data Management}

- Data collected will be entered into a registered electronic database by research staff who are separate to the clinical care of the participants.

- Hardcopies of participants' data will be stored in a secure office. 
- The electronic database will include the study identification number but no directly identifying data such as medical record number, date of birth or personal address.

- The de-identified database will be backed up on a server at Royal Prince Alfred Hospital.

- Data linking identifying details to the study number will be kept at a separate location in a locked filing cabinet.

- At study completion, data will be kept in a locked filing cabinet, and de-identified electronic data will be kept on a portable device such as a USB drive in a separate secure location at Royal Prince Alfred Hospital.

- All electronic data will be checked for accuracy by a second member of the research team and any apparent data entry errors will be discussed by the primary investigators and investigated/corrected as required.

\section{Discussion}

The correct identification of mothers who are colonised with GBS near the time of birth is a key aspect of determining which babies are most at risk of EOGBSD. Currently in Australia the two forms of screening for GBS are universal culture-based screening and the risk-factor approach. The transience of GBS combined with the unpredictable date of delivery can lead to the poor predictive value of a GBS culture swab performed antenatally. While more babies develop EOGBSD in settings that use the risk-factor approach meaning that some hospitals refuse to adopt this method. A recent integrative review by Braye et al. [19] makes a strong case for reviewing and updating GBS screening of pregnant women in Australia.

If intrapartum screening is to be beneficial, the PCR technology needs to: offer high accuracy for identifying GBS status; be user-friendly for the healthcare practitioner; require low maintenance; be cost-neutral or cost-effective compared to other screening methods; lead to a decrease in EOGBSD cases; and, ensure that fewer women unnecessarily receive prophylactic antibiotics [15].

Point-of-care intrapartum PCR screening for GBS has the potential to more accurately identify women who are GBS positive near the time of birth and the literature indicates that point-of-care PCR technology has been implemented in various settings due to high sensitivity, specificity and cost-effectiveness. Further potential benefits of point-of-care PCR screening include: GBS unknown women will now be able to be tested at the onset of labour in the labour ward; term women who rupture their membranes before onset of labour can be screened for GBS at that time rather than offered an induction of labour based on a GBS positive status at 35 - 37 weeks gestation as is current policy in some settings; and, a cost-saving is possible in that women having an elective caesarean section will no longer require GBS screening unless they rupture their membranes.

GBS screening by Xpert GBS (Cepheid) has been found to be "cost-neutral" when compared to universal antenatal culture-based screening [15]. However 
this outcome was based on screening by vaginal swab rather than a combined vaginal and perianal swab which would be the method used in our study. Ultimately further research is needed into the accuracy and cost-effectiveness of point-of-care PCR screening for GBS in different settings such as Australia.

\section{Research Ethics Approval}

Approval will be sought from the Ethics Review Committee of the Sydney Local Health District, Sydney, Australia.

\section{Protocol Amendments}

If modification to the study protocol is necessary, then permission will be sought from the ethics committee and the changes will be described in the final report.

\section{Confidentiality}

All the information collected from the study will be treated confidentially, and only the researchers will have access to it. The electronic database will be de-identified and stored at a different location to codes linking identifying data to study identification numbers. The electronic database will be on Microsoft Access, password-protected, and only accessible by research staff.

\section{Conflicts of Interest}

The researchers have no competing interests to declare.

\section{Significance}

This project will be the first in Australia to investigate the accuracy and cost-effectiveness of PCR by Xpert GBS (Cepheid) screening for GBS [18].

\section{References}

[1] Manning, M.L., Pfeiffer, J. and Larson, E.L. (2016) Combating Antibiotic Resistance: The Role of Nursing in Antibiotic Stewardship. American Journal of Infection Control, 44, 1454-1457. https://doi.org/10.1016/j.ajic.2016.06.023

[2] Cars, O., Molstad, S. and Melander, A. (2001) Variation in Antibiotic use in the European Union. Lancet, 357, 1851-1853.

https://doi.org/10.1016/S0140-6736(00)04972-2

[3] Fleming-Dutra, K.E., Hersh, A.L., Shapiro, D.J., Bartoces, M., Enns, E.A. and File, T.M. (2016) Prevalence of Inappropriate Antibiotic Prescriptions among US Ambulatory Care Visits, 2010-2011. Journal of the American Medical Association, 315, 1864-1873. https://doi.org/10.1001/jama.2016.4151

[4] Gavino, M. and Wang, E. (2007) A Comparison of a New Rapid Real-Time Polymerase Chain Reaction System to Traditional Culture in Determining Group B Streptococcus Colonization. American Journal of Obstetrics and Gynacology, 197, 388e1-388e4.

[5] Kwatra, G., Cunnington, M., Merrall, E., Adrian, P.V., Ip, M., Klugman, K.P., Tam, W.H. and Madhi, S.A. (2016) Prevalence of Maternal Colonisation with Group B 
Streptococcus: A Systematic Review and Meta-Analysis. The Lancet Infectious Diseases, 16, 1076-1084. https://doi.org/10.1016/S1473-3099(16)30055-X

[6] Garland, S.M. and Kelly, N. (1995) A Study of Group B Streptococcus in Brisbane; the Epidemiology, Detection by PCR Assay and Serovar Prevalence. Medical Journal of Australia, 162, 413-417.

[7] Colbourn, T. and Gilbert, R. (2007) An Overview of the Natural History of Early Onset Group B Streptococcal. Early Human Development, 83, 149-156. https://doi.org/10.1016/j.earlhumdev.2007.01.004

[8] Jeffery, H.E. and Lahra, M.M. (1998) Eight-Year Outcome of Universal Screening and Intrapartum Antibiotics for Maternal Group B Streptococcal Carriers. Pediatrics, 101, E2. https://doi.org/10.1542/peds.101.1.e2

[9] The Royal Australian and New Zealand College of Gynaecologists and Obstetricians (2003) Maternal Group B Streptococcus in Pregnancy: Screening and Management, College Statement, Revised March 2016, RANZCOG, Melbourne.

[10] Centers for Disease Control and Prevention (2010) Prevention of Perinatal Group B Streptococcal Disease Revised Guidelines from CDC, CDC Atlanta.

[11] Yancey, M.K., Schuchat, A., Brown, L.K., Ventura, V.L. and Markenson, G.R. (1996) The Accuracy of Late Antenatal Screening Cultures in Predicting Group B Streptococcal Colonization at Delivery. Obstetrics and Gynecology, 88, 811-815. https://doi.org/10.1016/0029-7844(96)00320-1

[12] Royal College of Obstetricians and Gynaecologists (2012) The Prevention of Early-onset Neonatal Group B Streptococcal Disease, Green-Top Guideline No36. 2nd Edition, RCOG, London.

[13] Kurz, E. (2015) Routine Culture-Based Screening versus Risk-Based Management for the Prevention of Early Onset Group B Streptococcus Disease in the Neonate: A Systematic Review. JBI Database of Systematic Reviews and Implementation Reports, 13, 206-246. https://doi.org/10.11124/jbisrir-2015-1876

[14] El Helali, N., Nguyen, J.C., Ly, A., Giovangrandi, Y. and Trinquart, L. (2009) Diagnostic Accuracy of a Real-Time Polymerase Chain Reaction Assay for Universal Intrapartum Group B Streptococcus Screening. Clinical Infectious Diseases, 49, 417-423. https://doi.org/10.1086/600303

[15] El Helali, N., Giovangrandi, Y., Guyot, K., Chevet, K., Gutmann, L. and Durand-Zaleski, I. (2012) Cost and Effectiveness of Intrapartum Group B Streptococcus Polymerase Chain Reaction Screening for Term Deliveries. Obstetrics and Gynecology, 119, 822-829. https://doi.org/10.1097/AOG.0b013e31824b1461

[16] Bujang, M.A. and Adnan, T.H. (2016) Requirements for Minimum Sample Size for Sensitivity and Specificity Analysis. Journal of Clinical and Diagnostic Research for doctors, 10, YE01-YE06.

[17] Devane, D., Begley, C., Clarke, M., Horey, D. and Oboyle, C. (2007) Evaluating Maternity Care: A Core Set of Outcome Measures. Birth, 34, 164-172. https://doi.org/10.1111/j.1523-536X.2006.00145.x

[18] NSW Health (2016) Maternal-Group B Streptococcus (GBS) and Minimisation of Neonatal Early-Onset GBS Sepsis. Ministry of Health, Sydney.

[19] Braye, K., Ferguson, J., Davis, D., Catling, C., Monk, A. and Foureur, M. (2018) Effectiveness of Intrapartum Antibiotic Prophylaxis for Early-Onset Group B Streptococcal Infection: An Integrative Review. Women and Birth, 31, 244-253.

https://doi.org/10.1016/j.wombi.2017.10.012 